Journal of the Magnetics Society of Japan Vol. 15 Supplement, No. S2 (1991)

(C) 1991 by The Magnetics Society of Japan

\title{
NOISE CHARACTERIZATION OF PERPENDICULAR MEDIA
}

\author{
R. S. INDECK, M. N. JOHNSON, Guo MIAN, J. R. HOINVILLE and M. W. MULLER
}

Department of Electrical Engineering, Washington University, St. Louis, MO 63130-4899 USA

\begin{abstract}
We have applied our precision disk tester and noise measurement procedures to perpendicular media. We look at the randomness of dc media noise from write to write, quantify the deterministic component of this noise, and investigate the head noise and dc track edge noise of perpendicular heads and media. These results are compared with those obtained for longitudinal media. We observe lower correlation coefficients than those for longitudinal media, suggesting that there is more randomness associated with the reversal mechanisms involved in our experiment. We also see that the dc track edge noise is imperceptibly small using our testing operation, implying that double-layered perpendicular dc track edges are of a different form than those of thin film longitudinal single-layered media.
\end{abstract}

\section{INTRODUCTION}

As recording densities are increased, media noise plays an increasingly important role in determining the limits of a recording system. If there is a correspondence between crystalline microstructure and magnetic microstructure, smaller bit cells will have an increasingly larger noise signal associated with them. If track edges do not scale with all other dimensions, track edge noise will become more prominent as the track densities increase. In order to develop the next generation magnetic recording media these noise features must be characterized and understood. Methods of investigating media noise phenomena experimentally have been developed and used to study longitudinal media[1],[2],[3]. We will present noise measurements for perpendicular media, and we will compare our results to those obtained using longitudinal media.

Our results rely upon experiments using our precision disk tester. The key to these results has been a magnetic synchronization technique developed to measure signals from a known place on the disk. Unlike other methods which use two heads, this method employs only a single head, thus reducing the differential thermal drifting associated with two separate sliders or fixture members.
Conjoined with this method is the development of an analysis of the correlation coefficient between two time-averaged waveforms. This gives us extreme spatial precision as well as an analytical tool to differentiate between the random (timevarying or write-to-write) component of the signal and that which is time or magnetic state invariant. The deterministic component may be a measure of the dependence of the magnetic noise signal on the medium's crystalline microstructure.

We have also used our system for the measurement of track edge noise in perpendicular media. Track edge noise[4], which in perpendicular media may be similar to transition noise, may not scale with the dimensions of the recording system. Track edge noise may therefore affect the ability of a medium to support ultra-high density recording. In perpendicular media the magnetic structure of a track edge is different from that in longitudinal media, where the magnetization is constrained to lie in the plane of the magnetic film.

\section{EXPERIMENT}

In an effort to separate the deterministic medium noise from random noises such as head noise and electronics noise, an averaging procedure 
is used; by averaging repeatedly measured waveforms, the random component of the signal approaches zero, while the "fixed" component remains non-zero. Others[5][6] have used a twin head scheme for determining the position of one head relative to the disk by encoding positioning information on the disk surface, and reading this with a position head which is fixed relative the head used for measurements. Since our measurements involve extensive movement across the disk surface, we require a positioning scheme involving only one head. This is described here[7]. First a dc saturated region is obtained by setting the head current for media saturation and slowly moving the energized head across the rotating medium. Next a magnetic transition is written on the disk, positioned in the middle of the erased band, as shown in Figure 1. When the head passes over this

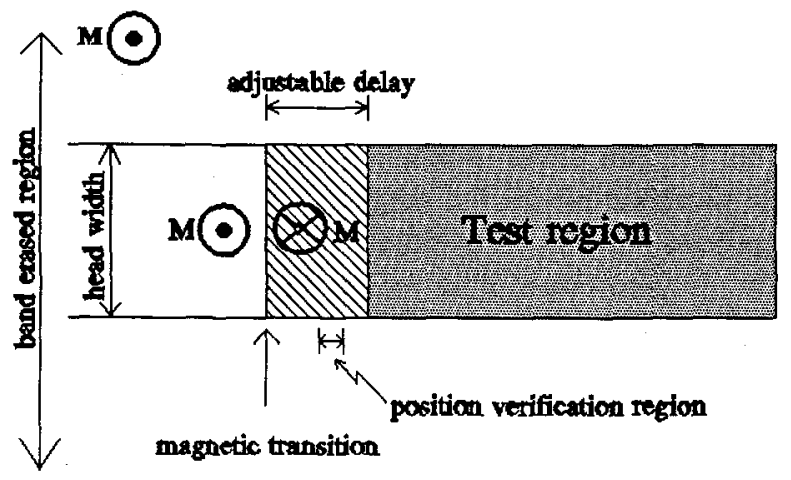

Figure 1. Magnetic synchronization schematic.

transition, the output is a pulse, which is then used as the trigger signal. Immediately following this magnetic trigger, a small region is reserved for determining the on-track position performance of the head during the experiment. An adjustable delay in the write electronics determines how far past the magnetic trigger the test region is

located. Our oscilloscope is set to trigger on the magnetic transition, then delay the necessary amount to read in the test region.
Using the magnetic trigger as a reference, we write and read our data with great precision at a fixed place on the disk. In this single head method, with one head being used for both trigger and $\mathrm{read} / \mathrm{write}$, many problems, such as alignment and thermal expansion of different head holders, are avoided.

Using the magnetic synchronization technique described above and a correlation analysis[8][9], we can determine with high precision the head's position on the disk relative to the trigger. The readback waveforms are averaged up to 2048 times. Since both the phase and the amplitude of the electronic and the head noises are randomly distributed, these noises are virtually eliminated after averaging such a large number of times. Since the data are read from the same place on the disk each time, the noise caused by medium inhomogeneities will remain after averaging. We have achieved high resolution of the head off-track displacement and down-track timing using medium noise correlation analysis.

\section{RESULTS AND DISCUSSION}

\section{Linear Off-Track Correlation}

We have employed our testing system for the determination of the correlation of the noise across a track. If we assume that the noise across the track is uncorrelated, then a linear relationship between the correlation coefficient and head displacement can be shown to exist. We have explored this for longitudinal and perpendicular media, and we do see, within the accuracy of our tester, that the noise is indeed linear, thus implying that the noise across the track is uncorrelated[8],[9]. The cross correlation coefficient versus distance off-track for our double-layered perpendicular media is shown in Figure 2. We see a linear relationship indicating that perpendicular media possesses noise which is uncorrelated across the track.

\section{Cross correlation coefficient analysis}

Since our tester provides us with accurate and steady positioning control, we can look at the same position on the disk after changing its state through an intervening write. The amount of 


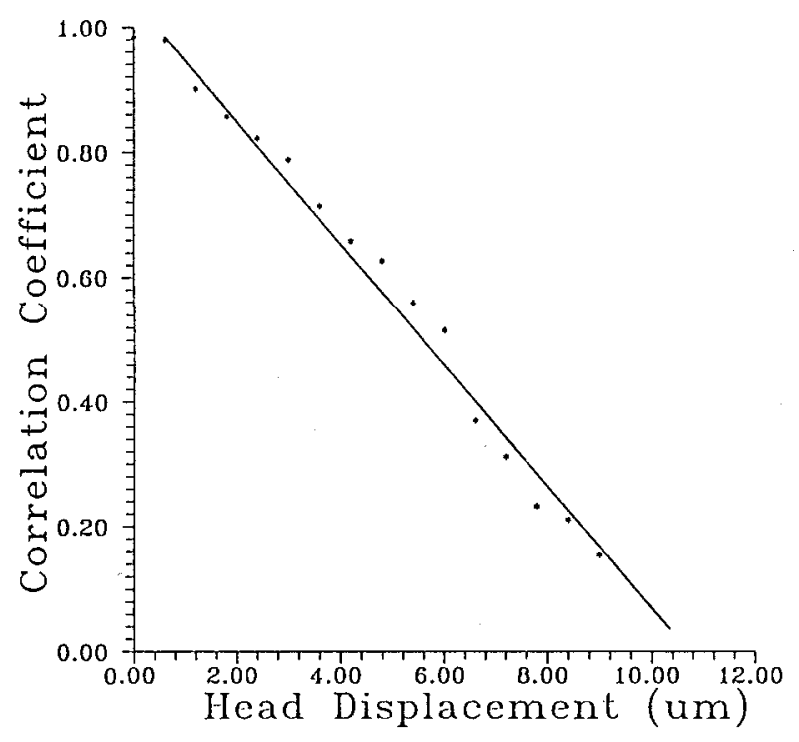

Figure 2. Correlation coefficient versus head displacement.

correlation between waveforms tells us about the degree to which the medium's resulting magnetization is deterministic, say resulting from medium microstructure, and how much signal is derived from random processes. For example, if a section of track is read with no intervening write we would expect the normalized cross correlation coefficient to be unity provided that there are no random components involved in the read process. Non-repeatable noise, such as electronics noise, bearing runout, flying height variations, and the like, can be effectively removed through averaging as described earlier. In our system, we obtain coefficients of about 0.99 between two waveforms when we read the same undisturbed region on the disk, and we routinely use this effect as a check of the system's performance (syncing accuracy and track misregistration).

We apply this technique to investigate noise by writing a dc erased track and then comparing the noise output of a small portion of the track (say 100 micrometers) to the same portion of track after writing. If the noise were completely deterministic, then no matter what intervening writes were done, when the medium was dc erased again the resulting coefficient would be unity. Continuing with the assumption that the noise is completely deterministic, we might expect that the noise from a negatively dc erased track would be exactly opposite that of the positive dc noise, being its mirror image and possessing a correlation coefficient of negative unity. Figure 3 shows the

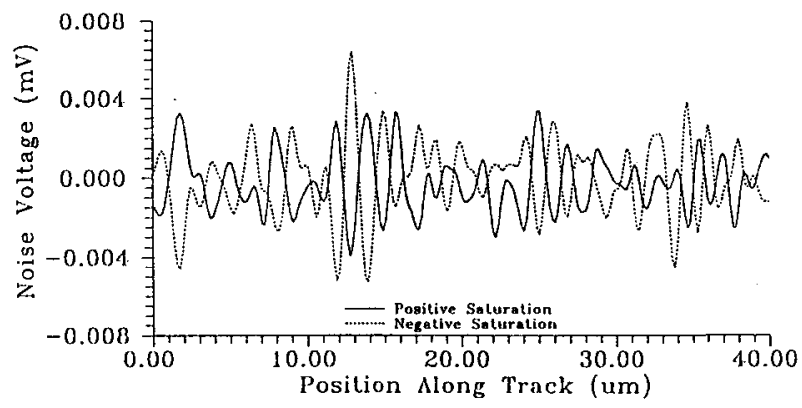

Figure 3. Noise voltage versus position.

output waveforms of a small portion of a dc erased track for both positive and negative erasure. Some mirror imaging can clearly be seen for the voltages suggesting that a portion of the output is indeed deterministic. We have attempted to quantify this degree of correlation by investigating the correlation coefficient. The correlation coefficients as a function of write events can be seen in Figure 4.

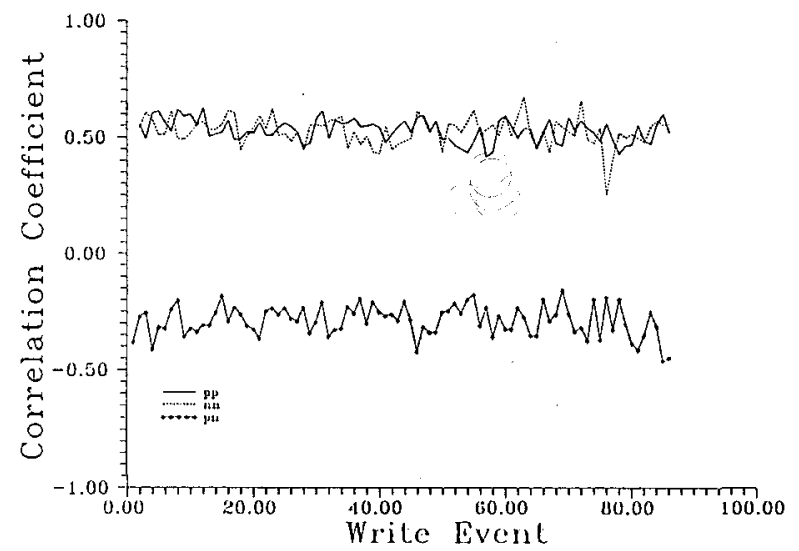

Figure 4. Correlation coefficient versus write event number. 
We see that the numerical value of the coefficient for successive positive writes with an intervening negative write (or similarly successive negative writes with intervening positive writes) is roughly 0.55 . The coefficient obtained between the states of positive and negative saturation is about -0.30 . These results indicate that a fair degree of deterministic processes exist in the perpendicular media. However, most longitudinal media investigated had a much higher degree of correlation $(0.93,-0.89)[2]$ than that of the perpendicular media tested. This may suggest that the reversal mechanisms and the demagnetizing fields involved in perpendicular switching are more random in nature than those of longitudinal media. The lower degree of correlation was found in all of the perpendicular media tested. It should be noted that the signal-to-noise ratios for the perpendicular headmedium combinations were roughly the same as those for the longitudinal heads and media tested.

Included in the experiments was an analysis to separate the deterministic and random components from the output[2]. Figure 5 shows some spectra from two perpendicular media. These media were quite different in structure as can be seen by the different spectral curves, and were dramatically different from longitudinal media which contained much more energy in the lower spatial frequencies.

Another valuable measurement technique is demonstrated in the density plot of Figure 6.

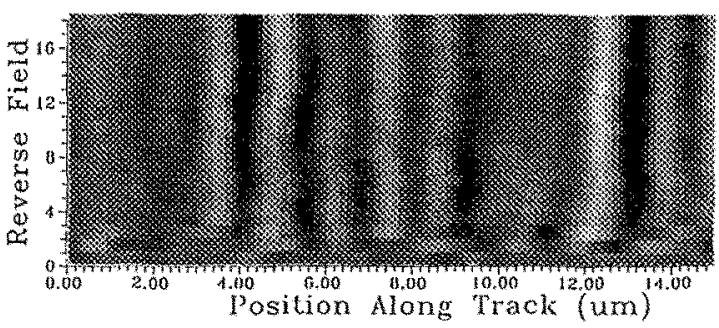

Figure 6. Density plot showing noise voltages as a function of position and applied reverse write field.

Starting from positive dc erasure, this experiment shows the noise voltage waveform change as the write current is gradually made more and more

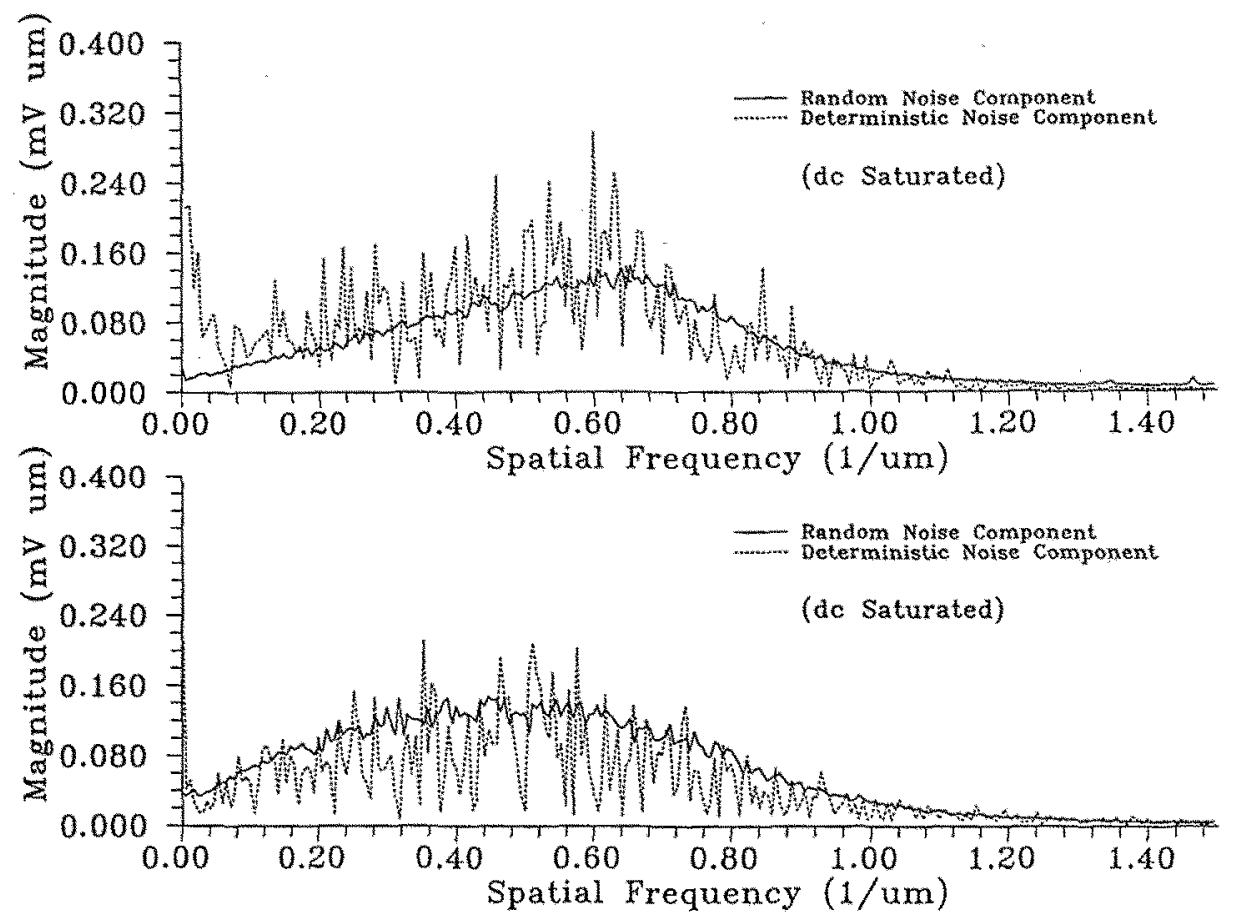

Figure 5. Noise spectra from two different dc erased perpendicular media. 
negative. We can see that the voltage waveforms on either extreme virtually mirror image one another as these correspond to positive and negative dc erasure. The transition from positive to negative magnetization occurs at a reverse field of about 2 in the plot of Figure 6 (reverse field units are arbitrary). How the noise transforms between these two states tells us something about the reversal process in the media. For example, the squarer the hysteresis loop the sharper the transition in applied field (in write head current) will be. The magnitude also describes the kind of reversal which takes place -- a medium which breaks up into large regions would have a larger noise power than one that did not. This has been described for longitudinal media as the dc demagnetized region[10]. We have investigated several longitudinal media as well as perpendicular media and we find very strikingly different results. The longitudinal media behaved as described, with the noise power increasing dramatically as the magnetization went through the demagnetized state. The perpendicular media did not show a significant increase in the noise power but the magnetization did indeed change as can be seen in the density plot of Figure 6.

\section{Track edge measurements}

Another striking difference between our measurements of perpendicular media and longitudinal media is the characterization of the noise arising from track edges. While there is clearly visible evidence of track edge noise in all of our longitudinal media tested, even those which had low dc noise[4], there was no perceptible noise from the dc track edges obtained in perpendicular media. This phenomenon is not totally unexpected. The magnetization of a track edge in longitudinal thin film media is that of an uncharged wall or line dipole (to a first order approximation), if a continuous, in-plane magnetization is assumed. This situation is entirely different from that of the perpendicular medium where we assume nearly decoupled grains.
Head noise and sensitivity randomness

The last test performed on the perpendicular head-media combination was the effect of head noise and sensitivity variations from write to write. The experiment was performed by reading and storing an output voltage for a section of the disk, writing another section of the disk which disturbs the head magnetization, then reading the original section and comparing the output with that stored. If the head returns to its original configuration, i.e. sensitivity and noise character, then the correlation coefficient would be unity. Any deviation from this is a randomness of the head behavior imposed by the changes from the write operation. We have seen significant random components for longitudinal thin film heads (as much as $8 \%$ ) but the randomness in the heads used in our perpendicular experiments were much smaller, only about two percent. This may be due to the interaction between the head and the medium, specifically the soft magnetic underlayer. The re-magnetization effects may be large enough to place the head into the same state on every read, independent of the history of the write head. Another factor which may affect the head's random nature is the structure of the head such as the main pole thickness and composition.

\section{CONCLUSIONS}

We have applied many noise measurement techniques to double-layered perpendicular media and single-sided thin film perpendicular write heads. Using an extensive correlation analysis we have determined that the media noise can be described as being uncorrelated across the track (to the spatial resolution of our tester), and "mirror imaging" of the dc noise voltage was seen between negative and positive erasure. Comparing the results to those obtained from longitudinal media we note the following differences: the randomness of a dc erased state is larger for perpendicular media; and track edge noise was imperceptible in our experimentation with perpendicular media. These methods may be combined with other techniques to characterize media and assist in the development of the next generation magnetic recording systems. 


\section{ACKNOWLEDGEMENTS}

We gratefully thank S. C. Reising and A. Chandra of the Magnetics Research Laboratory at Washington University for their contributions to this work, and to Censtor, IBM, and the other institutions who have supplied materials for this research. R. S. Indeck is supported in part by The IBM Faculty Development Award and the National Science Foundation Presidential Young Investigator Award (ECS-8957148). Portions of this work were performed under NSF grant MIP-9020883.

\section{REFERENCES}

[1] E. J. Yarmchuck, IEEE Trans. MAG-22, 877 (1986).

[2] J. R. Hoinville, M. W. Muller and R. S. Indeck, to be submitted to IEEE Trans MAG-27.

[3] R. S. Indeck, Guo Mian and M. W. Muller, to be submitted to IEEE Trans MAG-27.

[4] See for example R. S. Indeck, S. C. Reising, J. R. Hoinville and M. W. Muller, J. Appl. Phys., 69, 4721 (1991).

[5] S. Ohara and I. Sato, IEEE Trans. Magn., MAG-23, 2380 (1987).

[6] M. J. Vos, Y. Tanaka and J. H. Judy, IEEE Trans Magn., MAG-26, 2149 (1990).

[7] For detailed information see S. C. Reising, 1991 Washington University Master's Thesis and Washington University Technical Report WUEE-91-102 (1991).

[8] Guo Mian, R. S. Indeck and M. W. Muller, Japn. J. Appl. Phys., Part 2, 30-8B (1991) (To be published).

[9] Guo Mian, R. S. Indeck and M. W. Muller, IEEE Trans. MAG-27 (1991) (In press).

[10] H. Aoi, M. Saitoh, M. Nishiyama, R. Tsuchiya and T. Tamura, IEEE Trans. MAG-22, 895 (1988). 